\title{
Mother-infant relationship and its Predictors
}

\author{
Masoomeh Darvishvand ${ }^{1}$, Zahra Bostani Khalesi ${ }^{2}$, Seyedeh Marzieh Rahebi ${ }^{2}$ \\ ${ }^{1}$ Student Research Committee, Guilan University of Medical Sciences, Rasht, Iran \\ ${ }^{2}$ Social Determinants of Health Research Center, Guilan University of Medical Sciences, Rasht, Iran
}

\begin{abstract}
Objective: Creating attachment between mother and infant is an extremely sensitive process in the postpartum period. In this regard, understanding the predictive features of the mother-infant relationship (MIR) can help health care providers in designing and implementing MIR enhancement interventions. Therefore, we ran this study to determine the degree of attachment between mother and infant and its predictors.

Methods: This was a cross-sectional study on 406 mothers referred to comprehensive healthcare centers in Rasht. We used the multi-stage cluster random sampling. Our data collection tools included: a data collection form to determine the predictors of maternal and infant attachment, which was designed by researchers and the standard MIRscale. The significance level of the test was considered less than $5 \%$.

Results: The mean MIR score was $12.7 \pm 8.1$. The highest score of attachment between mother and infant was in the area of risk of infant harassment and the lowest score was in the field of the mother-infant bond. The results of the logistic regression test showed that the MIR scores were statistically significant with spouse age, spouse education, number of deliveries, and breastfeeding problems $(p<0.05)$.

Conclusions: The findings showed that there was a significant relationship between MIR and spouse age, spouse education, number of deliveries, and breastfeeding problems. Therefore, attention to these issues should be considered in planning to improve the MIR.
\end{abstract}

Keywords: relationship, mother, infant

\section{INTRODUCTION}

The mother-infant relationship (MIR) is an extremely sensitive process in the postpartum period (Dubber et al., 2015). The most important effect of a useful MIR is the healthy social and emotional development of infants to occur (Kliegman \& St Geme, 2019). The healthy relationship between mother and infant promotes personality traits such as curiosity, social ability, cooperation, independence, and higher levels of self-esteem in infants, and directs the child's behavior in future social relationships (Numan \& Young, 2016). Without this relationship, the child is at increased risk for psychological and social difficulties (Scatliffe et al., 2019). Indeed longitudinal studies have reported that an infant's ability to form and maintain healthy relationships throughout life may be significantly impaired by having an insecure relationship with the mother (Toosi et al., 2014). Researchers have reported the children who suffered a form of insecure relationship in their infancy are at risk of reduced hippocampal development (memory impairments), reduced growth in the left brain hemisphere (depression), and increased limbic system sensitivity (anxiety disorders) (Korja et al., 2012). The prevalence of disorder of the MIR (rejection of the child, delays or lack of emotional response, hostility, feelings of irritability, and aggressive impulse) has been reported in up to $25 \%$ of the mothers (Barrett et al., 2012). MIR disorders can lead to an increase in the child's risk of developing psychopathology during adulthood (Leyh et al., 2016). In addition, the lack of relationship between mother and infant leads to several individuals and social consequences, including aggression, depression, anxiety (Cambonie et al., 2017), poorer social functioning, violent behavior toward others, and delinquency (Nordahl et al., 2020).

Various factors may bring about MIR disorders. MIR may be influenced by infant-related factors such as late birth, physical problems (Nakano et al., 2019), restlessness, breastfeeding, birth weight, current infant weight (Galeshi et al., 2016), infant gender, infant preferred sex, and time of breastfeeding onset (Dezvaree et al., 2016). Also, factors related to parents, especially mothers, such as social support network (Tichelman et al., 2019), physical illness, postpartum anxiety, depression, other psychological problems related to the mother, type of recent delivery, preparation for pregnancy (Śliwerski et al., 2020), gestational age at delivery, duration of hospitalization, maternal age, maternal occupation, maternal education, history of abortion, history of stillbirth, a tendency to recent pregnancy and marital status, should all be considered (Muzik et al., 2013). In Iran, there are no mother-infant relationship services and this limits studies on MIR (Torshizi \& Sharifzadeh, 2013). Despite the evidence that there are considerable benefits of MIR, there are still gaps in understanding why many mothers have difficulties initiating and maintaining a healthy relationship with their infants. Given the important effects of MIR on the future health and development of the infant, it is important to investigate MIR-predicting factors in order to early identify and support mothers at heightened risk for relationship problems. Therefore, we decided to conduct a study to determine the extent of MIR and its predictors.

\section{MATERIAL AND METHODS}

It was a cross-sectional analytical study on 406 mothers referred to the comprehensive health centers in Rasht. The inclusion criteria were: interest in participating in the study, ability to read and write, age 16 years and older, no history of mental or incurable illness, having an infant aged 8 weeks and older (up to 12 months), living with a spouse, having one, healthy and at term baby, non-hospitalization of the mother due to postpartum complications, non-death or hospitalization of the infant in the intensive care unit, unpleasant events, such as death or hospitalization of a family member. When choosing when to evaluate birth after childbirth, it is important to note that a parent's perception of parenthood may change for the better during the first few months after birth. We believe about 8 weeks after birth, a woman's perception of herself as a mother is widely established and defined as a relatively real perception of her child (Gibbs et al., 2018). Therefore, the study was postponed until 8 weeks after delivery. Samples were collected by multi-stage cluster sampling. First, 42 centers and comprehensive health bases under the auspices of Guilan University of Medical Sciences in Rasht were 
classified into four clusters based on 4 geographical regions of north, south, east, and west. Then, using a table of random numbers, 12 centers and bases were selected. Inside each center, referring mothers were selected by available criteria if they met the inclusion criteria.

The data collection tool in this study was a questionnaire consisting of two parts. The first part of the data collection form was to determine the predictive factors of the MIR, which was designed by researchers to try and evaluate all the factors in related studies in this form. In this section, we assessed factors related to the personal-social characteristics of the mother (age, education, occupation, smoking, marital satisfaction, the level of support of the spouse in childcare, the level of family support in child support, marital status, physical illness, ethnicity), individual-social characteristics of the spouse (age, education, occupation, average monthly income), individual characteristics of the child (age, gender, sex satisfaction of the child, breastfeeding, time of breastfeeding, infant birth weight, the current weight of the baby, breastfeeding problems), reproductive history (whether or not the pregnancy was wanted, infertility history, history of abortion and fetal death, number of abortions, stillbirth history, history of pregnancy complications, type of delivery, gestational age at delivery, length of hospital stay).

The second part included the "mother-infant-postpartum-relationship" scale, design and psychometrics by Brockington. The scale has 25 questions in four areas: Mother-infant relationship disorders (12 questions), rejection and pathological anger ( 7 questions), infant-focused anxiety (4 questions), and incipient abuse (2 questions). These questions are scored on a Likert scale from always to never with a score of 0-5. Positive answers are scored from zero (always) to five (never). The scores range from zero to 125 and a high score indicates a high relationship between the mother and infant (Brockington et al., 2006). At first, after having the permission of the main designer of the scale, we translated it into Persian, using the open translation method by two people who were fluent in translating medical texts. Then, the translations were compared and the questions were matched in terms of meaning and concept, and the best options were selected, finally, a Persian version of this tool was prepared. Then, to ensure that the Persian translation corresponded to the original text and that the sentences of the Persian text were accessible, the translated version was translated back into the original language by another translator fluent in English who had not previously seen the original questionnaire. At this stage, the English versions were compared and after reviewing and making the necessary corrections, a single version of the English scale was obtained. Content validity was used to determine the validity of the questionnaire. For this purpose, in order to determine the content validity, a questionnaire was provided to 12 specialists and faculty members, and their opinions were applied. To determine the reliability of the instrument, the questionnaire was completed twice through a ten-day interval by 20 samples with the inclusion criteria, and we determined the Cronbach's alpha coefficient for the entire instrument, of 0.89 , and for the domains, it went from 0.72 to 0.91 . To establish the instrument's internal consistency we used the split-half method, and the internal consistency of the questionnaire was 0.6.

The researcher collected information after obtaining the permission of the ethics committee and after obtaining written permission from the Vice Chancellor for Research and Technology of Guilan University of Medical Sciences and the provincial health center, and stating the research objectives to the mothers who met the inclusion criteria.
Data analysis was performed using the $\mathrm{Spss}_{16}$. Descriptive statistical indicators were used to determine the MIR score in general and by domains, and we used the independent t-test and ANOVA to compare the MIR scores in terms of demographic variables. Moreover, we used the Pearson's correlation coefficient to examine the correlation of scores with quantitative variables; and the multiple linear or logistic regression models to determine the MIR predictive. The significance level of the test was considered less than $5 \%$.

\section{RESULTS}

The majority of mothers were in the age group of 25 to 29 years, with an average of 29.7 years. Eighty-five percent of the mothers were native, $35.2 \%$ had university education, $89.9 \%$ were housewives, $76.8 \%$ of the mothers were satisfied with their economic status, $78.58 \%$ of the mothers were happy with their marriages, and $98.5 \%$ of the mothers were pleased with their infant's gender. The age of the majority of the infants $(42.6 \%)$ was four months and less, with an average of 5.96 months. $58.6 \%$ of the infants were boys and $41.4 \%$ were breastfed.

The majority had spouses in the age group of 30 to 39 years, with an average of 33.7 years, $33 \%$ had a spouse with a university degree and $87.6 \%$ were self-employed. In terms of satisfaction with the economic situation, they were happy with it (Table 1 ).

Among the mothers, $77.6 \%$ had planned their pregnancies. Also, the average weight of infant at birth was $3370.20 \mathrm{~g}$ and the average current weight of the infant was $7216.16 \mathrm{~g}$. The average number of pregnancies was 1.71 , the average number of deliveries was 1.52 , and the number of abortions was 1.23 . Among the mothers, $66 \%$ gave birth by cesarean section; $94.1 \%$ of the mothers had the support from their spouses and $86.5 \%$ had the support of relatives and family to care for the children.

The mean total score of mother-infant relationship (0125 ) in the studied samples was $12.7 \pm 8.1$, and only $2 \%$ of the studied samples had severe disorders. The highest percentage of disorders were in the MIR disorders (12.3\%) (Table 2).

Univariate analysis between MIR and its dimensions with a variation of mother's age $(p=0.018)$, ethnicity $(p=0.03)$, age of spouse $(p=0.17)$, education of spouse $(p=0.031)$, marital life satisfaction $(p=0.005)$, planned pregnancy $(p=0.001)$, were statistically significant.

According to the Spearman's correlation coefficient, the relationship score had a significant correlation with maternal age $(p=0.014$ and $r=0.122)$, spouse age $(p=0.001$ and $r=0.169)$, number of pregnancies $(p<0.001$ and $r=0.236)$, and the number of deliveries $(p<0.001$ and $r=0.264)$.

We used a logistic regression model to determine relationship predictors. In the initial model, all variables with $(p<0.1)$ were included in the univariate analysis. Among these variables, the variables concerning spouse age $(p=0.02)$, spouse education $(p=0.014)$, presence of breastfeeding problems $(p=0.05)$, and the number of deliveries $(p<0.001)$ were determined as predictors of MIR. Spouse age and number of deliveries increased the likelihood of relationship disorders, with odds ratios of 1.08 and 6.62 , respectively. In this way, for every one year of increased spouse age, the chance of relationship disorder increased by 1.08 . Also, with each delivery, the chance of relationship disorder increases by 6.62 . But, with each increase in the spouse's educational level, the likelihood of relationship disorder, decreased by $38 \%$ (odds ratio 0.62 ). Breast feeding problems also increased the likelihood of relationship disorders by 2.2 times (odds ratio: 2.22) (Table 3). 
Table 1. Distribution of participants according to demographic features.

\begin{tabular}{|c|c|c|c|}
\hline \multicolumn{3}{|c|}{ Variable } & $(N=406) \%$ \\
\hline \multirow{3}{*}{ Age, Mean (y) } & \multicolumn{2}{|l|}{ Mother } & 29.7 \\
\hline & \multicolumn{2}{|l|}{ Father } & 33.7 \\
\hline & \multicolumn{2}{|l|}{ Infant } & 5.96 \\
\hline \multirow{8}{*}{ Education Status } & \multirow{4}{*}{ Mother } & Primary or Secondary School & $18(4.4)$ \\
\hline & & High School & $58(14.3)$ \\
\hline & & Diploma & $187(46.1)$ \\
\hline & & University education & $143(35.2)$ \\
\hline & \multirow{4}{*}{ Father } & Primary or Secondary School & $30(7.4)$ \\
\hline & & High School & $63(15.5)$ \\
\hline & & Diploma & $179(44.1)$ \\
\hline & & University education & $134(33)$ \\
\hline \multirow{4}{*}{ Employment Status } & \multirow{2}{*}{ Mother } & Housewives & $365(89.9)$ \\
\hline & & Employed & $41(10.1)$ \\
\hline & \multirow{2}{*}{ Father } & Self-employed & $315(87.6)$ \\
\hline & & Employed & $91(22.4)$ \\
\hline \multirow{3}{*}{\multicolumn{2}{|c|}{ Monthly Income (million) }} & $<1$ & $35(8.62)$ \\
\hline & & $1-2$ & $102(25.12)$ \\
\hline & & $>2$ & $269(66.26)$ \\
\hline \multirow{2}{*}{\multicolumn{2}{|c|}{ Life satisfaction }} & Yes & $328(80.78)$ \\
\hline & & No & $78(19.21)$ \\
\hline \multirow{2}{*}{\multicolumn{2}{|c|}{ Marital satisfaction }} & Yes & $319(78.58)$ \\
\hline & & No & $87(21.42)$ \\
\hline \multirow{2}{*}{\multicolumn{2}{|c|}{ Gender of the baby }} & Girl & $168(41.4)$ \\
\hline & & boy & $238(58.6)$ \\
\hline \multirow{6}{*}{ Substance use } & \multirow{2}{*}{ Mother } & No & $404(99.5)$ \\
\hline & & Cigarettes & $2(0.5)$ \\
\hline & \multirow{4}{*}{ Father } & No & $307(75.62)$ \\
\hline & & Cigarettes & $77(18.96)$ \\
\hline & & Opium & $1(0.24)$ \\
\hline & & Alcohol & $21(5.18)$ \\
\hline
\end{tabular}

Table 2. Distribution of maternal - infant relationship score, total and sub-scales.

\begin{tabular}{|c|c|c|c|c|}
\hline \multirow{2}{*}{\multicolumn{2}{|c|}{ Maternal - Infant Relationship }} & \multirow{3}{*}{$\frac{\mathbf{N}(\%)}{365(87.7)}$} & \multicolumn{2}{|c|}{ 95\% CI } \\
\hline & & & Lower limit & Upper limit \\
\hline \multirow{2}{*}{ Mother-infant relationship disorders (0-60) } & Without disorder & & 84.2 & 90.6 \\
\hline & With disorder & $50(12.3)$ & 9.4 & 15.8 \\
\hline \multirow{2}{*}{ Rejection and Pathological Anger (0-30) } & Without disorder & $406(100)$ & 0 & 0 \\
\hline & With disorder & $0(0)$ & 0 & 0 \\
\hline \multirow{2}{*}{ Infant-Focused Anxiety (0-20) } & Without disorder & $403(99.3)$ & 98 & 99.8 \\
\hline & With disorder & $3(0.7)$ & 0.2 & 2 \\
\hline \multirow{2}{*}{ Incipient Abuse (0-10) } & Without disorder & $405(99.8)$ & 98.9 & 100 \\
\hline & With disorder & $1(0.2)$ & 0 & 1.1 \\
\hline \multirow{3}{*}{ Total score (0-125) } & Without disorder & $368(90.6)$ & 87.5 & 93.2 \\
\hline & Weak disorder & $37(9.1)$ & 6.6 & 12.2 \\
\hline & Sever disorder & $1(0.2)$ & 0 & 1.1 \\
\hline
\end{tabular}


Table 3. Association between mother-infant relationship and other variables using multivariate logistic regression analysis.

\begin{tabular}{|c|c|c|c|c|c|c|}
\hline \multirow{2}{*}{ Variables } & \multirow{2}{*}{$\begin{array}{l}\text { Parameter } \\
\text { estimation }\end{array}$} & \multirow{2}{*}{ SE } & \multirow{2}{*}{$p$-value } & \multirow{2}{*}{ OR } & \multicolumn{2}{|c|}{ 95\% CI } \\
\hline & & & & & Lower limit & Upper limit \\
\hline Age of spouse & 0.073 & 0.031 & 0.02 & 1.076 & 1.012 & 1.145 \\
\hline Education of spouse & 0.476 & 0.194 & 0.014 & 0.621 & 0.425 & 0.909 \\
\hline Breastfeeding problems & 0.797 & 0.409 & 0.052 & 2.218 & 0.995 & 4.947 \\
\hline Number of deliveries & 0.964 & 0.274 & 0.001 & 6.622 & 1.532 & 4.486 \\
\hline Fixed values & 3.367 & 1.412 & 0.017 & 0.034 & & \\
\hline
\end{tabular}

$\mathrm{SE}=$ Standard Error

OR=Odd Ratio

$\mathrm{CI}=$ Confidence Interval

\section{DISCUSSION}

This study aimed at establishing the degree of relationship between mother and infant and its predictors. Findings showed that the highest MIR score was related to the risk of infant harm and the lowest score was related to MIR disorders. Moreover, based on the logistic regression test results, the MIR score with spouse age, spouse education, number of deliveries, and breastfeeding problems were statistically significant.

There was a significant relationship between maternal age and MIR in a univariate analysis, but this relationship was not significant in the logistic regression model. In these regards, the results of a study by Rossen et al. (2017) showed that older maternal age predicted a poor relationship between mother and infant, and this is probably due to the fact that older women suffer from disruptions in their jobs or delayed pregnancy, and are thus less inclined to play a maternal role. The results of the present study did not show a significant relationship between maternal occupation and MIR. However, Abbas et al. stated that mothers who worked part-time or chose other options had a lower relationship during pregnancy than the mothers who worked full-time. He believes that women who work part-time or have chosen other options may be less likely to report postpartum relationship (Abbas et al., 2018). One of the reasons for this contradiction between the results of the present study and the study from Abbas et al. is that in our study, the majority of mothers were housewives and only $11 \%$ of them were employed. The results of other studies were in line with the results of the present study.

In our study, there was a significant relationship between the age of the spouse and the relationship with the mother. Other studies have not examined the effects of this variable. In the present study, the results showed a significant relationship between spouse education and MIR. In this way, the higher the education of the spouse, the higher the MIR score. However, the results of the study by Galeshi et al. (2016) did not show a significant relationship between spouse education and the relationship score (Galeshi et al., 2016). The reasons for this discrepancy can be pointed to the fact that due to cultural differences and the fact that the majority of the samples in the present study had diplomas and higher education, spouse education was shown as an influential variable on the MIR.

In the present study, there was a significant relationship between breastfeeding problems and MIR. In this way, having a problem with breastfeeding reduces the MIR score. In these regards, the results of a study by Rossen et al. showed that having two or more breastfeeding problems is one of the MIR predictors. Rossen also stated that there are several theoretical mechanisms that suggest that breastfeeding increases the MIR. However, there is only a few empirical studies to support this, and the results are uncertain. It is possible that breastfeeding may be associated with maternal and infant relationship through oxytocin secretion (Rossen et al., 2017).

"Oxytocin production in mothers continues with milk production after birth, and the secretion of this hormone is in response to infant behaviors such as sucking, clinging to the mother, facial expressions and infant sounds," says Galbally et al. (2011). They stated that oxytocin has an important role in developing and regulating the parent-infant relationship in humans (Galbally et al., 2011).

The mean score of the relationship was $1.8 \pm 7.12$. In studies conducted in Iran, the average relationship scores were very different (Dadipoor et al., 2014). In a study by Galeshi et al. (2016), in Tabriz, 85.2\% of mothers with MIR were impaired. In another similar study by Alaei et al. in Tehran, the average relationship score was $56.59 \%$ (Alaei et al., 2006). On the other hand, the average relationship score obtained in the study held by Moghadam Hosseini in Mashhad, yielded a high difference when compared to other studies, estimated to be $97.48 \%$ (Moghadam Hosseini, 2011).

\section{CONCLUSION}

The primary aim of our study was to extend knowledge on the mother-infant relationship processes during the first year of life. This study aimed to fill a salient gap in the literature regarding these processes during the first year of life. The findings showed that spouse age and education, presence of breastfeeding problems, and the number of deliveries were predictors of the mother-infant relationship. These results have important methodological implications in the assessment of mother-infant relationship processes.

Findings from these studies led the authors in both cases to recommend that future research explore perceptions of the mother in other types of family structures (Single, Cohabiting, and Married Families).

\section{ACKNOWLEDGMENTS}

This paper is taken from the MSc thesis on midwifery training with the ethics code (No. IR.GUMS.REC.1397.172). The researchers sincerely appreciate the cooperation and support of the Department of Research and Technology of the Guilan University of Medical Sciences. We also appreciate the support and assistance of all the pregnant women who participated in this study.

\section{CONFLICT OF INTEREST}

No potential or any conflict of interest was reported. 


\section{Corresponding author:}

Zahra Bostani Khalesi

Shahid Beheshti Nursing and Midwifery School

Guilan, Iran.

E-mail: z_bostani@yahoo.com

\section{REFERENCES}

Abbas SI, Turkistani MH, Al-Gamdi AA, Alzahrani SA, Alzahrani AA, Helmy FF. Factors associated with postnatally maternal-infant attachment in taif, Saudi Arabia. Saudi J Health Sci. 2018;7:127-31. DOI: 10.4103/sjhs.sjhs_50_18

Alaei F, Shahabi M, Mohammadi R, Alavi-Majd H. Maternal attachment behaviors at first neonatal visit in a hospital affiliated to Shahid Beheshti Medical University. J Nurs Midwifery Shahid Beheshti Univ Med Sci. 2006;15:31-9.

Barrett J, Wonch KE, Gonzalez A, Ali N, Steiner M, Hall GB, Fleming AS. Maternal affect and quality of parenting experiences are related to amygdala response to infant faces. Soc Neurosci. 2012;7:252-68. PMID: 21943083 DOI: $10.1080 / 17470919.2011 .609907$

Brockington IF, Fraser C, Wilson D. The Postpartum Bonding Questionnaire: a validation. Arch Womens Ment Health. 2006;9:233-42. PMID: 16673041 DOI: $10.1007 /$ s00737006-0132-1

Cambonie G, Muller JB, Ehlinger V, Roy J, Guédeney A, Lebeaux C, Kaminski M, Alberge C, Denizot S, Ancel PY, Arnaud C; OLIMPE study writing group. Mother-infant interaction assessment at discharge and at 6 months in a French cohort of infants born very preterm: The OLIMPE study. PLoS One. 2017;12:e0188942. PMID: 29216238 DOI: $10.1371 /$ journal.pone.0188942

Dadipoor S, Rajaei M, Ahmadi Kani Golzar A, Karimi T, Eghbali R, Hesam A, Moradabadi AS. Investigating the degree of mother-infant relationship among the visitors of Bandar Abbas hospitals. Pars J Med Sci. 2014;12:17-23.

Dezvaree N, Alaeekarahroudi F, KhanaliAgan L, TalebiGhane E. The Mother-Newborn's Attachment and its Related Factors in Mothers of Hospitalized Preterm Neonates. J Health Care. 2016;17:340-9.

Dubber S, Reck C, Müller M, Gawlik S. Postpartum bonding: the role of perinatal depression, anxiety and maternal-fetal bonding during pregnancy. Arch Womens Ment Health. 2015;18:187-95. PMID: 25088531 DOI: 10.1007/ s00737-014-0445-4

Galbally M, Lewis AJ, Ijzendoorn Mv, Permezel M. The role of oxytocin in mother-infant relations: a systematic review of human studies. Harv Rev Psychiatry. 2011;19:1-14. PMID: 21250892 DOI: 10.3109/10673229.2011.549771

Galeshi M, Mirghafourvand M, Alizadeh-Sharajabad F, Sanaati F. Predictors of Mother-Child Bonding. Hayat. 2016;22:13-26.

Gibbs BG, Forste R, Lybbert E. Breastfeeding, Parenting, and Infant Attachment Behaviors. Matern Child Health J. 2018;22:579-88. PMID: 29388115 DOI: 10.1007/s10995$018-2427-z$

Kliegman RM, St Geme J. Nelson Textbook of Pediatrics. 21st ed. Philadelphia: Elsevier; 2019.
Korja $R$, Latva $R$, Lehtonen $L$. The effects of preterm birth on mother-infant interaction and attachment during the infant's first two years. Acta Obstet Gynecol Scand. 2012;91:164-73. PMID: 22007730 DOI: 10.1111/j.1600-0412.2011.01304.x

Leyh R, Heinisch C, Behringer J, Reiner I, Spangler G. Maternal Attachment Representation and Neurophysiological Processing during the Perception of Infants' Emotional Expressions. PLoS One. 2016;11:e0147294. PMID: 26862743 DOI: 10.1371/journal.pone.0147294

Moghadam Hosseini V. Impact of Maternal Attachment Style on Mother to Infant Attachment. Qom Univ Med Sci J. 2011;5:87-91. Persian.

Muzik M, Bocknek EL, Broderick A, Richardson P, Rosenblum $\mathrm{KL}$, Thelen $\mathrm{K}$, Seng JS. Mother-infant bonding impairment across the first 6 months postpartum: the primacy of psychopathology in women with childhood abuse and neglect histories. Arch Womens Ment Health. 2013;16:29-38. PMID: 23064898 DOI: $10.1007 / \mathrm{s} 00737-012-0312-0$

Nakano M, Upadhyaya S, Chudal R, Skokauskas N, Luntamo $T$, Sourander A, Kaneko H. Risk factors for impaired maternal bonding when infants are 3 months old: a longitudinal population based study from Japan. BMC Psychiatry. 2019;19:87. PMID: 30849963 DOI: 10.1186/s12888-019-2068-9

Nordahl D, Rognmo K, Bohne A, Landsem IP, Moe V, Wang CEA, Høifødt RS. Adult attachment style and maternal-infant bonding: the indirect path of parenting stress. BMC Psychol. 2020;8:58. PMID: 32513300 DOI: 10.1186/s40359-020-00424-2

Numan M, Young LJ. Neural mechanisms of mother-infant bonding and pair bonding: Similarities, differences, and broader implications. Horm Behav. 2016;77:98-112. PMID: 26062432 DOI: 10.1016/j.yhbeh.2015.05.015

Rossen L, Hutchinson D, Wilson J, Burns L, Allsop S, Elliott EJ, Jacobs S, Macdonald JA, Olsson C, Mattick RP. Maternal Bonding through Pregnancy and Postnatal: Findings from an Australian Longitudinal Study. Am J Perinatol. 2017;34:80817. PMID: 28212591 DOI: $10.1055 / \mathrm{s}-0037-1599052$

Scatliffe N, Casavant S, Vittner D, Cong X. Oxytocin and early parent-infant interactions: A systematic review. Int J Nurs Sci. 2019;6:445-53. PMID: 31728399 DOI: 10.1016/j. ijnss.2019.09.009

Śliwerski A, Kossakowska K, Jarecka K, Świtalska J, Bielawska-Batorowicz E. The Effect of Maternal Depression on Infant Attachment: A Systematic Review. Int J Environ Res Public Health. 2020;17:2675. PMID: 32295106 DOI: 10.3390/ijerph17082675

Tichelman $E$, Westerneng $M$, Witteveen $A B$, van Baar $A L$, van der Horst $\mathrm{HE}$, de Jonge A, Berger MY, Schellevis FG, Burger $\mathrm{H}$, Peters LL. Correlates of prenatal and postnatal mother-to-infant bonding quality: A systematic review. PLoS One. 2019;14:e0222998. PMID: 31550274 DOI: 10.1371/journal.pone.0222998

Torshizi M, Sharifzadeh G. Maternal-fetal attachment and associated factors in pregnant women referred to Birjand health centers (2012). J Birjand Univ Med Sci. 2013;20:279-87.

Toosi M, Akbarzadeh M, Zare N, Sharif F. Comparison of the effects of attachment behavior and relaxation training on maternal fetal attachment and infant in prime pregnant women. J Fundam Ment Health. 2014;16:142-9. DOI: 10.22038/JFMH.2014.3271 